\title{
Eosinophils in corneas removed by penetrating keratoplasty
}

\author{
MICHAEL B LIMBERG, , CURTIS E MARGO, ${ }^{2}$ AND GARY H LYMAN ${ }^{2}$ \\ From the 'Lions Eye Research Laboratories, LSU Eye Center, Louisiana State University Medical Center \\ School of Medicine, New Orleans, and the ${ }^{2}$ Department of Ophthalmology, University of South Florida \\ Medical Center, Tampa, USA.
}

SUMMARY One hundred consecutively submitted corneas removed by penetrating keratoplasty were studied histologically to determine the frequency in which eosinophils are found. In 20 corneas eosinophils occurred in densities ranging from 0.2 to more than 50 per high power field (HPF), and accounted for $1.5 \%$ to $100 \%$ of the total leucocytes counted. More than half of the corneal specimens containing eosinophils, including six of seven corneas having the highest eosinophil concentrations, were failed grafts. Because the concentration of eosinophils was in many cases beyond that attributable to non-specific vascular permeability, the existence of eosinophil chemotaxic substances in the cornea is postulated.

The eosinophil has a poorly understood role in the inflammatory response and is typically associated with allergic disease and parasite infection. ${ }^{1-7}$ Other than an occasional mention of eosinophils in corneal tissue in such conditions as onchocerciasis, ${ }^{8}$ few corneal diseases are known to be associated with eosinophilic infiltrates. It has been our observation, however, that eosinophils are relatively common in corneas removed by penetrating keratoplasty, and that these cases encompass a wide variety of corneal disorders. Since the validity of this observation and its significance are unknown, we undertook a study to determine the conditions associated with the presence and frequency of eosinophils in the cornea.

\section{Material and methods}

One hundred consecutively submitted corneal buttons removed by penetrating keratoplasty were studied. Each cornea was fixed in formalin and examined with a dissecting microscope. Focal corneal abnormalities (for example, ulceration, scar, etc.) were included in the bisected meridian. Corneas were embedded in paraffin and stained with haematoxylin and eosin and with the periodic acid Schiff reagent. Superficial and $0.5 \mathrm{~mm}$ deep corneal cross sections were examined at $100 \times$ magnification for

Correspondence to Dr Michael B Limberg, LSU Eye Center, 136 South Roman Street, New Orleans, LA 70112, USA. the presence of eosinophils. Corneas in which one or more eosinophils were found were studied further.

The density of eosinophils was calculated from the total number of cells counted in five contiguous fields of $100 \times$ magnification. The ratio of eosinophils to other leucocytes was based on total cell counts from the same five fields. In all cases counts were initiated in areas of greatest eosinophil concentration. The final anatomical diagnosis was determined by histopathological findings and clinical diagnosis.

The causes of graft failure were evaluated retrospectively. When it was impossible to establish the cause with certainty, we correlated the most significant clinical or anatomical abnormalities. The diagnosis of recurrent herpes keratitis was based on clinical criteria, viral culture, or the presence of herpetic giant cells in the epithelium of the failed graft.

Medical records from each patient with corneal eosinophils were reviewed. The following information was obtained: age, sex, race, past medical history, systemic and topical medications, history of allergies, and past ocular history. Complete blood counts with leucocyte differential were recorded from blood specimens drawn the day prior to surgery. Culture results and corneal scrapings were reviewed in all cases of corneal ulcer.

The association of categorical variables was analysed by the $\chi^{2}$ method. 


\section{Results}

The final anatomical diagnoses of the 100 corneas removed by penetrating keratoplasty are listed in Table 1. The most common conditions necessitating

Table 1 Diagnosis in 100 corneal buttons

\begin{tabular}{ll}
\hline Diagnosis & Percent of cases \\
\hline Corneal oedema & $31 \%$ \\
$\quad$ Aphakic $(14 \%)$ & \\
Pseudophakic $(8 \%)$ & \\
$\quad$ Fuchs's dystrophy (9\%) & \\
Failed graft & $27 \%$ \\
Keratoconus & $15 \%$ \\
Corneal ulcer & $13 \%$ \\
Corneal scar & $6 \%$ \\
Miscellaneous* & $8 \%$ \\
\hline
\end{tabular}

${ }^{*}$ Herpes simplex keratitis (3), ocular pemphigoid (1), keratitis sicca (1), ocular leprosy (1), aniridia (1), and exposure keratopathy (1).

Table 2 Diagnosis of 20 corneas with eosinophils

\begin{tabular}{lc}
\hline Diagnosis & Number of cases \\
\hline Graft failure & 12 \\
Corneal ulcer & 3 \\
Corneal oedema (pseudophakic and aphakic) & 2 \\
Exposure keratopathy & 1 \\
Keratopathy of aniridia & 1 \\
Keratoconus & 1 \\
\hline
\end{tabular}

surgery were corneal oedema $(31 \%)$, graft failure $(27 \%)$, keratoconus $(15 \%)$, and non-healing corneal ulcer $(13 \%)$.

Twenty corneas had one or more eosinophils present (Table 2). The diagnosis in these cases included graft failure (12), non-healing corneal ulceration (3), corneal oedema (2), exposure keratopathy (1), ocular leprosy (1), keratopathy of aniridia (1), and keratoconus (1). The density of eosinophils expressed as the number of cells per high power field (HPF) ranged from 0.2 to more than 50 . The average number of eosinophils counted per cornea was $29 \cdot 5$. Eosinophils represented $1.8 \%$ to $100 \%$ of the total leucocytes in 14 corneas (Table 3). In six corneas differential counts could not be performed because necrosis prevented accurate identification of cells. Interestingly, eosinophils tended to be more resistant than other leucoctyes to the effects of cellular degeneration. There was no correlation between the density of eosinophils and their ratios to other inflammatory cells. Three of the 20 patients had abnormal blood count differentials consisting of greater than $4 \%$ eosinophils $(7 \%, 7 \%$, and $11 \%)$. In none of these patients were there any other systemic conditions to account for the peripheral eosinophilia.

Eleven of the 27 failed grafts in this study had eosinophils, and six of the seven corneas with the greatest concentration of eosinophils were failed grafts. While $27 \%$ of the initial 100 corneas were failed grafts, $55 \%$ of the corneas containing eosinophils were graft failures $(p<0 \cdot 001)$. The conditions

Table 3 Cases with corneal eosinophils

\begin{tabular}{|c|c|c|c|c|}
\hline & $\begin{array}{l}\text { Case } \\
\text { no.* }\end{array}$ & $\begin{array}{l}\text { Final } \\
\text { diagnosis }\end{array}$ & $\begin{array}{l}\text { Density } \\
\text { (no. eosinophils } \\
\text { per HPF) }\end{array}$ & $\begin{array}{l}\text { Prevalence } \\
\text { (no. eosinophils } \\
\text { per total leucocytes } \\
\times 100)\end{array}$ \\
\hline \multirow[t]{11}{*}{ Failed grafts } & 94 & Pseudomonas ulcer & $2 \cdot 8$ & $10 \%$ \\
\hline & 91 & Proteus ulcer & $>50$ & $\dagger$ \\
\hline & 52 & Serratia ulcer & $18 \cdot 2$ & $\dagger$ \\
\hline & 18 & Sterile ulcer & $1 \cdot 8$ & $\dagger$ \\
\hline & 53 & Fungal keratitis & $22 \cdot 0$ & $\dagger$ \\
\hline & 8 & Recurrent HSV keratitis & $16 \cdot 8$ & $28 \cdot 8 \%$ \\
\hline & 16 & Recurrent HSV keratitis & $7 \cdot 6$ & $32 \cdot 6 \%$ \\
\hline & 19 & Recurrent HSV keratitis & $4 \cdot 0$ & $62 \cdot 5 \%$ \\
\hline & 51 & Recurrent HSV keratitis & $12 \cdot 8$ & $100 \%$ \\
\hline & 43 & Graft failure & $2 \cdot 4$ & $50 \%$ \\
\hline & 45 & Graft failure & $1 \cdot 0$ & $33 \%$ \\
\hline \multirow[t]{9}{*}{ Native corneas } & 65 & Aphakic bullous keratopathy & $1 \cdot 2$ & $2 \cdot 1 \%$ \\
\hline & 80 & Aphakic bullous keratopathy & $0 \cdot 6$ & $13 \%$ \\
\hline & 1 & Pseudomonas ulcer & $19 \cdot 2$ & $100 \%$ \\
\hline & 35 & Pseudomonas ulcer & 0.8 & + \\
\hline & 42 & Sterile corneal ulcer & $3 \cdot 6$ & $56 \%$ \\
\hline & 27 & Exposure keratopathy & $0 \cdot 8$ & $1 \cdot 8 \%$ \\
\hline & 11 & Keratoconus & $0 \cdot 2$ & $\dagger$ \\
\hline & 46 & Ocular leprosy with perforation & 0.6 & $30 \%$ \\
\hline & 68 & Keratopathy of aniridia & $2 \cdot 2$ & $33 \%$ \\
\hline
\end{tabular}

*Twenty cases out of 100 consecutive, numbered cases examined. †Unable to differentiate leucocytes accurately. 
Table 4 Conditions associated with graft failures

\begin{tabular}{lll}
\hline Diagnosis & $\begin{array}{l}\text { Number of cases } \\
\text { with eosinophils } \\
(n=11)\end{array}$ & $\begin{array}{l}\text { Number of cases } \\
\text { without eosinophils } \\
(n=16)\end{array}$ \\
\hline Recurrent herpes simplex & 4 & 3 \\
Bacterial ulcer & 3 & 1 \\
Fungal ulcer & 1 & 0 \\
Sterile ulcer & 1 & 0 \\
Failure with no known & & \\
$\quad$ associated condition & 2 & 7 \\
Trauma & 0 & 2 \\
Exposure keratopathy & 0 & 1 \\
Keratitis sicca & 0 & 1 \\
Ocular hypertension & 0 & 1 \\
\hline
\end{tabular}

associated with graft failure are listed in Table 4. Three of the six grafts with large numbers of eosinophils occurred in patients who had had previous herpes simplex keratitis; two of these were 2 -year-old boys with previous primary herpes simplex keratitis. The other three occurred in patients having nonhealing corneal ulcers. The seventh cornea containing large numbers of eosinophils was from a patient who wore a contact lens and had an impending perforation due to pseudomonas infection. Although these seven cases appeared to have a substantially greater concentration of eosinophils, no statistically significant difference was observed between these groups because of the small number of cases involved $(\mathrm{p}>0 \cdot 10)$.

Eosinophils were found in stroma adjacent to blood vessels in all but one cornea. In that one case eosinophils were present in an ulcer bed. Eosinophils in the corneas with ulcers were more concentrated near vascular channels than near ulcer defects.

One of the 20 patients had a known allergy characterised as seasonal rhinitis. No association was found between the presence of corneal eosinophils and topical or systemic medication, systemic disease, race, or sex. The majority of patients in this series lived in the south-eastern part of the United States within a 200 -mile radius of New Orleans.

\section{Discussion}

One or more eosinophils were present in $20 \%$ of the corneas removed surgically in this series, a somewhat surprising observation, since corneal eosinophilia has rarely been reported. Detailed histopathological studies surveying corneal disease have not mentioned the eosinophil as an inflammatory component in the past. ${ }^{911}$ Our study shows that eosinophils are often involved in corneal inflammatory disease and in many cases accumulate in concentrations beyond those attributable to non-specific increased vascular permeability.
Corneal eosinophilia is not associated with obvious allergic or parasitic disease in the vast number of cases that ultimately require keratoplasty in the United States. Although in this study eosinophils were present in a variety of corneal conditions, they were noted significantly more often in vascularised failed grafts than in native corneas $(\mathrm{p}<0.001)$. While graft failures accounted for $27 \%$ of the initial cases included in the study, they comprised $55 \%$ of corneas that contained eosinophils. It is interesting that two corneas with high concentrations of eosinophils occurred in failed grafts from 2-year-old children diagnosed as having primary herpes simplex keratitis.

Eosinophils undergo chemotaxis to a wide variety of stimuli, including components of the complement system, histamine, bacterial products, and factors elaborated by stimulated lymphocytes. ${ }^{1-7}$ Although eosinophils are capable of phagocytosis, their more vital role is probably in dampening immediate hypersensitivity reactions by enzymatic degradation of chemotatic mediators. ${ }^{5}$ It is intriguing to speculate that this modulating effect may be operational in certain cases of corneal transplant rejection. One hundred and six years have passed since the ubiquitous eosinophil was identified by Ehrlich, and, although it is one of the most thoroughly described cells in the human body, its role in corneal disease remains totally obscure.

This work was supported in part by PHS grant EYO2377 from the National Eye Institute, National Institutes of Health, Bethesda, MD

\section{References}

1 Kazura JW. Protective role of eosinophils. In: Mahmoud AAF, Austen KF, eds. The eosinophil in health and disease. New York: Grune and Stratton, 1980: 231-48.

2 Goetzl EJ. Cellular properties of eosinophils: regulatory, protective, and potentially pathogenic roles in inflammatory states. In: Weissman G, Glynn LE, Houck JC, eds. Handbook of inflammation. New York: Elsevier/North Holland, 1980 2: 189-216.

3 Weller PF, Goetzl EJ. The human eosinophil. Roles in host defense and tissue injury. Am J Pathol 1980; 100: 791-820.

4 Beeson PB, Bass DA. The eosinophil. In: Smith LH, ed. Major problems in internal medicine. Philadelphia: Saunders, 1977: 14: 1-269.

5 Goetzl EJ, Wasserman SI, Austen KF. Eosinophil polymorphonuclear leucocyte function in immediate hypersensitivity. Arch Pathol 1975; 99: 1-12.

6 Ottesen EA, Cohen SG. The eosinophil, eosinophilia, and eosinophil related disorders. In: Middleton $\mathrm{E} \mathrm{Jr}$, Reed CE, Ellis EF, eds. Allergy, principles and practice. St Louis: Mosby, 1978 2: 584-632.

7 Gleich GJ. IgE antibodies, allergic inflammation and the role of the eosinophil. In: Suran A, Gery I, Nussenblatt R, eds. Immunology of the eye workshop III. Immunologic aspects of ocular disease: infection, inflammation, and allergy. Arlington: Information Retrieval Inc., 1981: 353-64. 
8 Paul PV, Zimmerman LE. Some observations on the ocular pathology of onchocerciasis. Hum Pathol 1970; 1: 581-94.

9 Yanoff M, Fine BS. Ocular pathology. 2nd Ed. Hagerstown: Harper and Row, 1982: 206-388.
10 Kurz GH, D'Amico RA. Histopathology of corneal graft failures. Am J Ophthalmol 1968; 66: 184-98.

11 Specer WH. Ophthalmic pathology. 3rd Ed. Philadelphia: Saunders, 1985: 229-388. 